\title{
Determinación de proteínas para la obtención de un material adhesivo a diferentes tiempos y temperaturas, a partir de la viruta de wet blue del proceso del curtido de pieles mediante hidrólisis alcalina
}

Determination of proteins to obtain an adhesive material at different times and temperatures, from the wet blue chip of the leather tanning process by means of alkaline hydrolysis

Miguel Valdez-Urbina ${ }^{\mathrm{a}}$, Liz González-Gutiérrez ${ }^{\mathrm{b}}$, Manuel Pariguana-Begazo ${ }^{\mathrm{c}}$,Roberto Lopez Guerra ${ }^{\mathrm{d}}$, Alex Dueñas-Gonza ${ }^{e}$.

aEscuela Profesional de Ingeniería Ambiental, Universidad Católica Santa María. Arequipa, Perú.

${ }^{\text {b} F a c u l t a d ~ d e ~ I n g e n i e r i ́ a ~ B i o t e c n o l o ́ g i c a ~ y ~ F a r m a c i a, ~ U n i v e r s i d a d ~ C a t o ́ l i c a ~ S a n t a ~ M a r i ́ a . ~ A r e q u i p a, ~ P e r u ́ . ~}$

'Facultad de Ingeniería Química Universidad de Barcelona, España.

${ }^{\mathrm{d}}$ Asociación de Curtiembre, ASPEMYCUR, Perú.

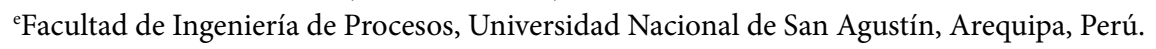

\section{INFORMACIÓN}

Historia del Artículo

Recepción: 21/07/2019

Revisión: 13/08/2019

Aceptación: 20/08/2019

\section{Palabras Clave}

Proteínas, hidrólisis, viruta, neutralización.

\section{Key Words}

Proteins, hydrolysis, chip, neutralization.

\section{DOI}

https://doi.org/10.35286/veritas. v20i2.246

\begin{abstract}
RESUMEN
El objetivo principal del presente estudio fue investigar la producción de un material adhesivo a partir de la viruta de wet blue producida a partir del proceso del curtido de pieles. La viruta seca de wet blue en una cantidad de 25 gr fue sometida a hidrólisis alcalina con $0.3 \mathrm{~N}, 0.4 \mathrm{~N}$ y $0.5 \mathrm{~N}$ de $\mathrm{NaOH}$, la hidrólisis se realizó a las temperaturas de $50^{\circ} \mathrm{c}, 60^{\circ} \mathrm{C}$ y $70^{\circ} \mathrm{C}$, se consideró 3 horas y 4 horas para detener la hidrólisis de la viruta, mediante la neutralización con $\mathrm{H} 2 \mathrm{SO} 4$ hasta llegar a un rango de $\mathrm{pH}$ entre 7 y 8 . En la caracterización de la viruta de wet blue se presentó $45.84 \%$ de proteína siendo en su mayoría colágeno y $23.21 \mathrm{mg} / \mathrm{kg}$ de cromo con un $50.42 \%$ de contenido de humedad. La mayor concentración de proteínas obtenida a partir del proceso de hidrólisis fue a las condiciones de $60^{\circ} \mathrm{C}$ de temperatura, $0.4 \mathrm{~N}$ de $\mathrm{NaOH}$ y 4 horas para la neutralización, con un promedio de $9.32 \mathrm{mg} / \mathrm{mL}$ de proteína, presentando diferencias altamente significativas $(\mathrm{p}<0.01)$ con los demás tratamientos. La concentración de proteínas obtenida a partir de las condiciones de hidrólisis óptima, indicarían ser un material adhesivo que podría ser utilizado en diferentes aplicaciones, pues la concentración de colágeno en pieles es mayor del $30 \%$ y en viruta supera el $39 \%$ de la composición.
\end{abstract}

\section{INTRODUCCIÓN}

La industria del cuero ha ido creciendo en el Perú sin ningún control por parte de los organismos fiscalizadores, esta industria se encarga de transformar pieles de animales en un material física y químicamente estable sometiéndolos a procesos secuenciales químicos y mecánicos, generalmente utiliza pieles y cueros como materia prima, que son los

Correspondencia:

Alex Dueñas-Gonza

aduenasg@unsa.edu.pe subproductos de la industria cárnica y de productos cárnicos (Ozgunay H,et al, 2007). En un mundo muy competitivo la preocupación básica de las empresas manufactureras es aumentar la satisfacción de sus clientes al mejorar constantemente su entrega y mantener la calidad en su mejor nivel, así también las empresas deben mantener sus costos y precios lo más bajo posible para poder competir con los demás manteniendo su rentabilidad (Mia MAS, et al 2017). A partir de $1000 \mathrm{~kg}$ de cuero crudo, se generan casi $850 \mathrm{~kg}$ como desechos sólidos en el procesamiento del cuero. Solo $150 \mathrm{~kg}$ de materia prima se convierten en cuero. La curtiduría genera una gran cantidad de desechos sólidos de la siguiente manera: descarnado, 50-60; afeitado de cromo, divisiones de cromo y polvo de pulir 35-40\%; recortes de piel, 5-7; y 
cabello, 2-5\% (Aftab Ali Shaikh, et al 2017). Un adhesivo es un material que se aplica a las superficies de los artículos para unirlos permanentemente mediante una unión adhesiva. proceso. Un adhesivo es una sustancia capaz de formar enlaces a cada uno de las dos partes cuando el objeto final consiste en dos secciones que están unidas (ASTM D 90782,1984). La preparación del adhesivo implica el estudio de los desechos sólidos que ayudarán al análisis de la estructura y los constituyentes químicos de estos desperdicios, en esta investigación se determinará la concentración de proteínas a partir de virutas de wet blue como material aglomerante.

\section{METODOLOGÍA}

\section{Obtención de la muestra}

La muestra correspondiente a residuos del proceso de raspado del cuero viruta de Wet Blue se obtuvieron de una empresa curtidora de pieles ubicada dentro del Parque industrial de Río Seco del distrito de Cerro Colorado de la ciudad de Arequipa- Perú.

\section{Determinación del tamaño de las virutas}

Las virutas de wet blue presentaron distintos tamaños dependiendo del tipo de cuero, se uniformizó la dimensión, utilizando tamizadores de $4 \mathrm{~mm}, 2 \mathrm{~mm}$ y de $1 \mathrm{~mm}$.

\section{Caracterización de la viruta de Wet Blue}

Para la caracterización se seleccionó una muestra inicial de $200 \mathrm{~kg}$ de viruta de Wet blue, mediante el método del cuarteo referente a residuos sólidos descrito en la guía metodológica para el desarrollo del estudio de caracterización de residuos sólidos municipales (EC-RSM) hasta la obtención de una muestra final de 100 grs., para analizar el porcentaje de proteínas, concentración de cromo y contenido de humedad.

\section{Hidrólisis alcalina}

Para la hidrólisis se utilizó un diseño DCR con arreglo factorial $3 \times 3 \times 2$ hidróxido de sodio a 3 concentraciones $0.3 \mathrm{~N}$ , $0.4 \mathrm{~N}$ y $0.5 \mathrm{~N}$, a tres temperaturas a las temperaturas de 50 ${ }^{\circ} \mathrm{C}, 60 \mathrm{C}^{\circ}$ y $70 \mathrm{C}^{\circ}$ a dos tiempos de reacción 3 horas y 4 horas.

\section{Determinación de las proteínas}

El porcentaje de proteínas, fue determinado mediante el método Kjeldahl para la concentración de nitrógeno, A.O.A.C. (Oficial Methods of Analysis 13 th Edition, 1984), y para el cálculo del porcentaje de proteína se utilizó el factor de conversión de 1,4 para transformar el nitrógeno total a proteína.

\section{Determinación del cromo}

La concentración de cromo de la viruta de Wet blue, fue determinado mediante el método de absorción atómica (EPA, 2007), previa digestión ácida con agua regia (mezcla de ácido clorhídrico $37 \%$ y ácido nítrico $69 \%$ en la proporción de 3:1)

\section{Determinación de la humedad}

Se pesó 100 grs. de viruta de wet blue en 6 crisoles las cuales se sometieron a $105^{\circ} \mathrm{C}$ en una estufa. Cada hora se sacó una crisol dejándolo en un desecador, la muestra fría se mide el peso, hasta que el peso no presenté una diferencia mayor a $2 \mathrm{mg}$ en un intervalo de $1 \mathrm{Hr}$. (norma INEN 565 NTE)

\section{RESULTADOS Y DISCUSIÓN}

\section{Determinación del tamaño de las virutas de Wet blue}

El tamaño de las virutas de Wet blue pasaron por tamizadores ( $4 \mathrm{~mm}, 2 \mathrm{~mm}$ y $1 \mathrm{~mm}$ ) obteniendo los siguientes resultados (tabla 1):

Tabla 1: Tamaño de las virutas del wet blue expresado en peso y porcentaje después del tamizado.

\begin{tabular}{cccc}
\hline Tamiz & $\begin{array}{c}\text { Retenido Parcial } \\
(\mathrm{gr})\end{array}$ & $\begin{array}{c}\text { Retenido acumula- } \\
\text { do (gr) }\end{array}$ & $\begin{array}{c}\text { Porcentaje } \\
(\%)\end{array}$ \\
\hline $4 \mathrm{~mm}$ & 26.34 & 26.34 & 26.34 \\
$2 \mathrm{~mm}$ & 53.89 & 80.23 & 53.89 \\
$1 \mathrm{~mm}$ & 14.44 & 94.67 & 19.77 \\
Fondo & 5.33 & 100.00 & 5.33 \\
\hline
\end{tabular}

Los resultados mostraron que el $53.89 \%$ de la viruta presentó en tamaño mayor a $2 \mathrm{~mm}$ pero menor de $4 \mathrm{~mm}$. Para que se produzca una hidrólisis en forma homogénea, el tamaño adecuado es menor de $1 \mathrm{~mm}$ (Campinas, 2017), sin embargo el $5.33 \%$ de la muestra de viruta de wet blue, para realizar una hidrólisis homogénea se realizó la adecuación de tamaño para uniformizar el proceso.

\section{Caracterización de la viruta de Wet blue}

Los resultados de la caracterización de la viruta de wet blue recogida de la curtiembre " Rogusbel S.A.C " realizados en los laboratorios de Control de Calidad de La Universidad Católica Santa María fueron los siguientes (tabla 2):

\section{Tabla 2: Caracterización de las virutas del wet blue}

\begin{tabular}{cc}
\hline Parámetro & Resultado \\
\hline Proteína & $45.84 \%$ \\
Cromo & $23.21 \mathrm{mg} / \mathrm{Kg}$ \\
Humedad & $50.42 \%$ \\
\hline
\end{tabular}

Se determinó en la caracterización de las virutas de wet blue que presentaron $45.84 \%$ de contenido de proteínas, el cromo se presentó en una concentración de $23.21 \mathrm{mg} / \mathrm{Kg}$ con una humedad de $50.42 \%$. 


\section{Hidrólisis alcalina de las virutas de wet blue}

Los resultados mostraron un efecto altamente significativo $(\mathrm{p}<0.01)$, en la concentración de proteínas extraídas de la viruta de wet blue por la aplicación de diferentes temperaturas, diferentes concentraciones de $\mathrm{NaOH}$ en diferentes tiempos de extracción con un efecto de interacción entre los mismos (tabla 3).

Tabla 3: : Efecto del tiempo, hidróxido de sodio y tiempo de la hidrólisis de la viruta del wet blue.

\begin{tabular}{|c|c|c|c|c|c|}
\hline FACTOR & $\begin{array}{l}\text { TIPO III DE } \\
\text { SUMA DE } \\
\text { CUADRADOS }\end{array}$ & $\mathrm{Gl}$ & $\begin{array}{c}\text { MEDIA } \\
\text { CUADRÁTICA }\end{array}$ & $\mathrm{F}$ & $\mathrm{P}$ \\
\hline TEMPERATURA & 2,813 & 2 & 1,407 & 66,929 & $\begin{array}{l}0,000 \ldots \\
(\mathrm{p}<0.01)\end{array}$ \\
\hline $\mathrm{NaOH}$ & 10,475 & 2 & 5,237 & 249,196 & $\begin{array}{l}0,000 \ldots \\
(\mathrm{p}<0.01)\end{array}$ \\
\hline TIEMPO & 36,820 & 1 & 36,820 & 1751,932 & $\begin{array}{l}0,000 \ldots \\
(\mathrm{p}<0.01)\end{array}$ \\
\hline $\begin{array}{c}\text { TEMPERATURA * } \\
\text { NaOH }\end{array}$ & 2,391 & 4 & 0,598 & 28,440 & $\begin{array}{l}0,000 \ldots \\
(\mathrm{p}<0.01)\end{array}$ \\
\hline $\begin{array}{l}\text { TEMPERATURA * } \\
\text { TIEMPO }\end{array}$ & 1,664 & 2 & 0,832 & 39,576 & $\begin{array}{l}0,000 \ldots \\
(\mathrm{p}<0.01)\end{array}$ \\
\hline $\mathrm{NaOH}{ }^{*}$ TIEMPO & 6,183 & 2 & 3,091 & 147,088 & $\begin{array}{l}0,000 \ldots \\
(\mathrm{p}<0.01)\end{array}$ \\
\hline $\begin{array}{l}\text { TEMPERATURA * } \\
\text { NaOH * TIEMPO }\end{array}$ & 1,347 & 4 & 0,337 & 16,028 & $\begin{array}{l}0,000 \ldots \\
(\mathrm{p}<0.01)\end{array}$ \\
\hline Error & 0,757 & 36 & 0,021 & & \\
\hline TOTAL & 62,449 & 53 & & & \\
\hline
\end{tabular}

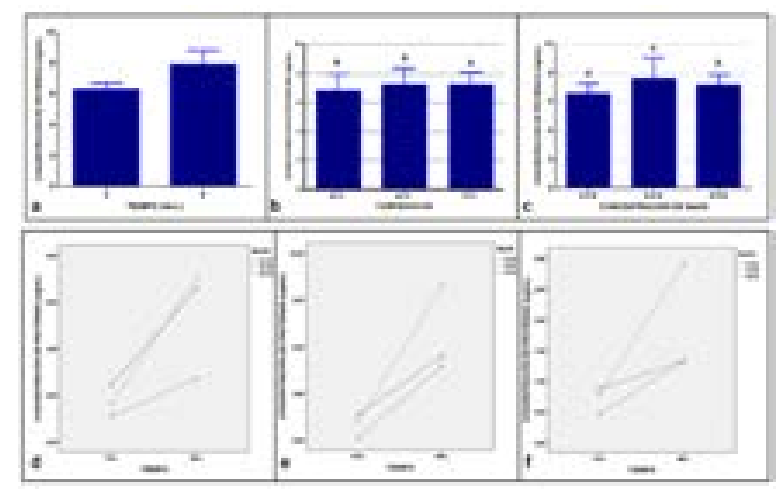

Fig. 1: Comparación de la concentración de proteínas, según concentraciones de $\mathrm{NaOH}$, temperaturas y tiempos de hidrólisis a) La comparación de los tiempos de hidrólisis demostró que un tiempo de 4 Hrs presentó mayor concentración de proteínas $7.94 \mathrm{mg} / \mathrm{mL}$. b) La prueba de comparación de Tukey muestra que las temperaturas de $60^{\circ} \mathrm{C}$ y $70^{\circ} \mathrm{C}$ presentaron mayor concentración de proteínas promedio con $7.27 \mathrm{mg} /$ $\mathrm{mL}$ y $7.28 \mathrm{mg} / \mathrm{mL}$ respectivamente. c) Se muestra que para la concentración de $4 \mathrm{~N}$ de $\mathrm{NaOH}$ se presentó la mayor concentración de proteínas con $7.62 \mathrm{mg} / \mathrm{ml}$. Los gráficos según temperatura de hidrólisis $\mathrm{d}\left(50^{\circ} \mathrm{C}\right), \mathrm{e}\left(60^{\circ} \mathrm{C}\right)$ y $\mathrm{f}\left(70^{\circ} \mathrm{C}\right)$ representan la interacción entre los factores concentración de $\mathrm{NaOH}$ y tiempo de hidrólisis de las virutas del wet blue, siendo la interacción con mayor concentración de proteínas concentración de $0.4 \mathrm{~N}$ tiempo de 4 horas de hidrólisis, la interacción con cual se obtuvo mayor concentración de proteínas promedio con $9.32 \mathrm{mg} / \mathrm{ml}$ para una temperatura de $60^{\circ} \mathrm{C}$.

\section{CONCLUSIÓN}

La mayor concentración de proteínas obtenida a partir del proceso de hidrólisis alcalina fue a las condiciones de $60^{\circ} \mathrm{C}$ de temperatura, con una concentración de $0.4 \mathrm{~N}$ de $\mathrm{NaOH}$ y 4 horas para la neutralización, con un promedio de $9.32 \mathrm{mg} / \mathrm{ml}$ de proteína. La concentración de proteínas obtenida a partir de estas condiciones de hidrólisis alcalina puede ser usado como material adhesivo que podría ser utilizado en diferentes aplicaciones.

\section{REFERENCIAS BIBLIOGRÁFICAS}

1. Adapted by Determination of Metals and trace elements in wáter and Wastes by Inductively Coupled Plasma Atomic Emission Spectrometry EPA method 2007.

2. Aftab Ali Shaikh, Amal Kanti Deb, Eama Akter, Tasnimul Ferdous, Md. Abu Sayid Mia. Resource addition to leather industry: Adhesive from chrome shaving dust. Journal of Scientific and Innovative Research 2017; 6(4): 138-141

3. A.O.A.C. Oficial Methods of Analysis 13 th Edition, 1984.

4. Atomic Emission Spectrometry EPA method 200.7

5. Mia MAS, Alam MNE, Rahman ML, Uddin MK. Footwear Industry in Bangladesh: Reduction of Lead time by using Lean Tools. JECET Sec. C, 2017; 6(3):251-259.

6. Modified from ASTM D 907-82, Standard Definitions of Terms Relating to Adhesives, published in Volume 15.06-Adhesives, Annual Book of ASTM Standards, 1984.

7. Ozgunay H, Colak S, Mutlu MM, Akyuz F. Characterization of Leather Industry Wastes.

8. Oficial Methods of Analysis 13 th Edition, 1984. 